\title{
Editorial
}

\section{Fuzzy Logic Applications in Control Theory and Systems Biology}

\author{
Sendren Sheng-Dong Xu, ${ }^{1}$ Hao Ying, ${ }^{2}$ Pablo Carbonell, ${ }^{3}$ \\ Ching-Hung Lee, ${ }^{4}$ and Wei-Sheng $\mathrm{Wu}^{5}$ \\ ${ }^{1}$ Graduate Institute of Automation and Control, National Taiwan University of Science and Technology, Taipei 10607, Taiwan \\ ${ }^{2}$ Department of Electrical and Computer Engineering, Wayne State University, Detroit, MI 48202, USA \\ ${ }^{3}$ University of Evry, iSSB, 91000 Evry, France \\ ${ }^{4}$ Department of Mechanical Engineering, National Chung Hsing University, Taichung 402, Taiwan \\ ${ }^{5}$ Department of Electrical Engineering, National Cheng Kung University, Tainan 701, Taiwan
}

Correspondence should be addressed to Sendren Sheng-Dong Xu; sdxu@mail.ntust.edu.tw

Received 2 May 2013; Accepted 2 May 2013

Copyright (C) 2013 Sendren Sheng-Dong Xu et al. This is an open access article distributed under the Creative Commons Attribution License, which permits unrestricted use, distribution, and reproduction in any medium, provided the original work is properly cited.

Fuzzy logic has shown itself to be a powerful design and analysis methodology in control theory, enabling the implementation of advanced knowledge-based control strategies for complex dynamic systems such as those emerging applications for systems and synthetic biology. This special issue on advanced fuzzy logic applications compiles seven exciting manuscripts. Four of the manuscripts discuss the effectiveness in applying fuzzy logic to solving control issues. The other three papers discuss the fuzzy retractions, fuzzification, and the fuzzy application in transportation systems.

S. Simani proposes the application of a data-driven fuzzy control design to a wind turbine benchmark model. The author discusses the fuzzy modeling and identification and suggests a fuzzy control approach for the adjustment of both the wind turbine blade pitch angle and the generator torque. H. Benítez-Pérez, A. Benítez-Pérez, J. Ortega-Arjona, and O. Esquivel-Flores discuss a way to tackle multiple time delays that are bounded and the dynamic response from real-time scheduling approximation. D. Krokavec and A. Filasová explore the new conditions suitable for design of a stabilizing output controller for a class of continuous-time Takagi-Sugeno nonlinear systems. N. V. Kolesov discusses the fault diagnosis using fuzzy interacting observers. Three different models of faults are considered, including structural changes, faults in the signal space, and faults in the parameter space. A. Paz, P. Maheshwari, P. Kachroo, and S. Ahmad discuss the estimation of performance indices for the planning of sustainable transportation systems by fuzzy logic. A. E. El-Ahmady and A. S. Al-Luhaybi study new types of fuzzy retractions of fuzzy open flat Robertson-Walker space. Finally, H. V. Kumbhojkar discusses the fuzzification of prime ideals of hemirings. Minimum imperative for proper fuzzification is suggested.

By compiling these articles, we hope to enrich our readers and researchers with respect to these particularly relevant, yet usually highly treatable, fuzzy logic applications.

Sendren Sheng-Dong Xu
Hao Ying
Pablo Carbonell
Ching-Hung Lee
Wei-Sheng Wu 

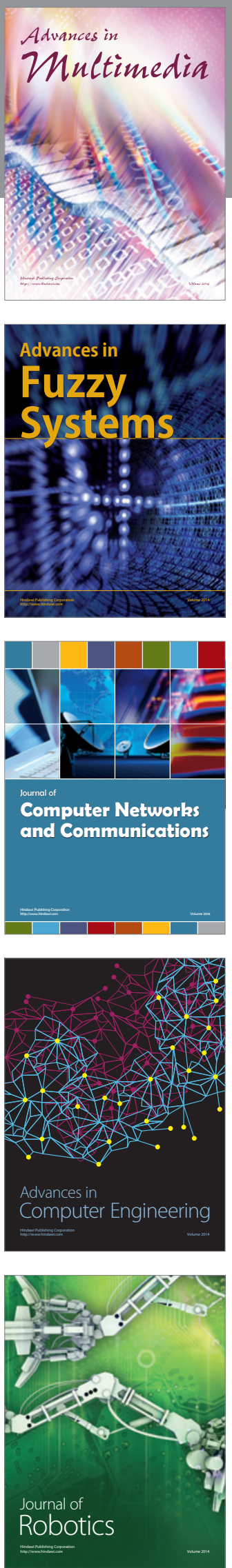

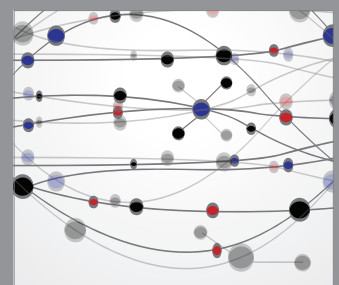

The Scientific World Journal
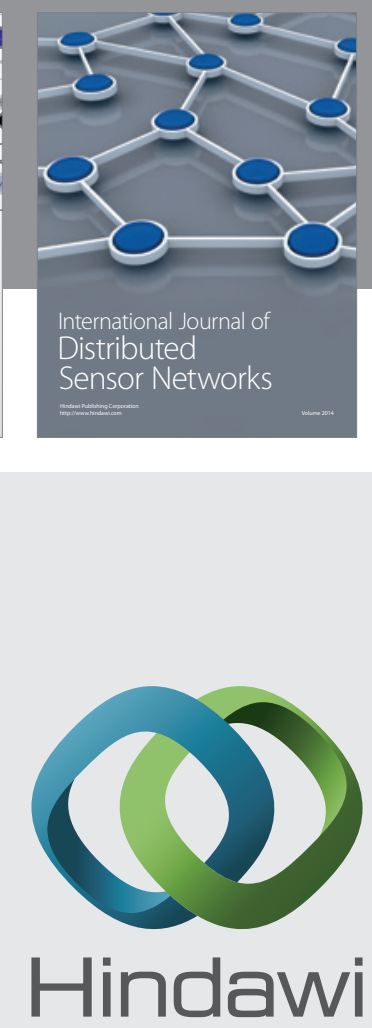

Submit your manuscripts at

http://www.hindawi.com
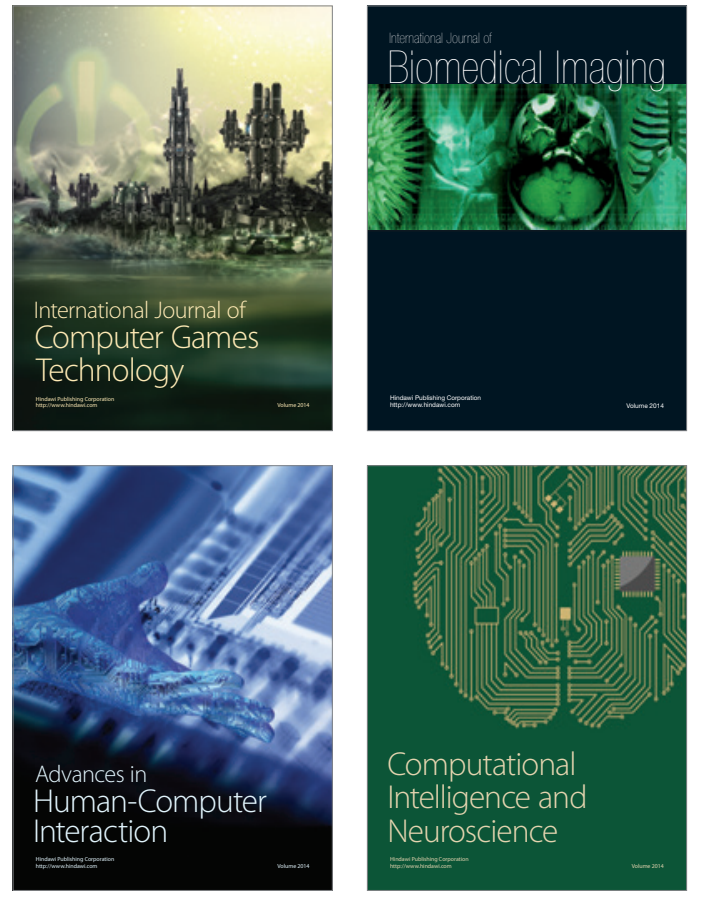
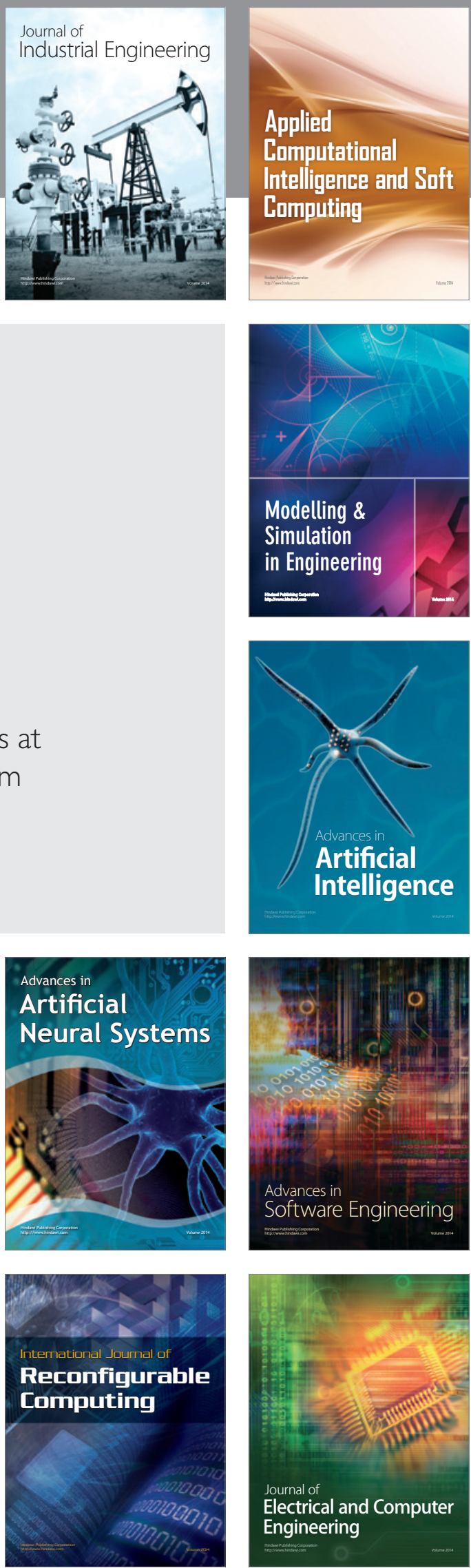